\title{
Consumo de sustancias estimulantes y drogas de abuso en el deporte: la experiencia italiana
}

\section{Use of stimulants and drugs of abuse in sport: the Italian experience}

\author{
Sabina Strano Rossi*; Maria Gabriella Abate*; Maria \\ Cristina Braganö; Francesco Botri ${ }^{*}$
}

\author{
*Laboratorio Antidoping Federazione Medico Sportiva Italiana. \\ Roma, Italia. \\ Enviar correspondencia a: \\ Sabina Strano Rossi. \\ Laboratorio Antidoping. Lg. G. Onesti, 1, 00197, Roma, Italia \\ E-mail sabina.stranorossi@gmail.com
}

\section{RESUMEN}

La incidencia de consumo de sustancias de abuso en el deporte puede ser un indicador útil de la difusión del fenómeno en la población juvenil. Muchas drogas han sido incluidas en la lista de sustancias prohibidas de la World Anti-Doping Agency (WADA) y son investigadas de rutina sobre las muestras analizadas en los controles antidoping.

Este estudio muestra los datos obtenidos en los controles efectuados en el laboratorio antidoping de Roma durante el periodo 2003-2007, sobre un total de 44781 muestras, con las metodologías desarrolladas por el laboratorio para la determinación de todas las sustancias prohibidas.

Los positivos encontrados en el periodo 2003-2007 varían entre el 1,1 y el $2 \%$ con una elevada incidencia de sustancias estimulantes y drogas de abuso. La sustancia más frecuentemente encontrada es el metabolito del cannabis, representando el 0,2-0,4\% del total; también se detecta frecuentemente la presencia de cocaina, con una incidencia de positividad del 0,1\%. Otros estimulantes encontrados fueron efedrinas, carfedon, modafinilo y anorexizantes (fendimetrazina y norfenfluramina) y ningún caso de anfetaminas o compuestos anfetaminosimiles fue detectado.

Estos datos pueden ser un indicador útil de la elevada incidencia del uso de cannabinoides y de cocaína por la población juvenil italiana, considerando una infraestimación del fenómeno debida al hecho que estos datos se refieren solo a controles efectuados durante la competición y a la propia población estudiada, atletas los cuales están sometidos a controles de salud y forma física.

Palabras clave: análisis de orina, drogas de abuso, controles antidoping, epidemiologia.

\section{ABSTRACT}

The incidence of the abuse of illicit drugs in sport may be a useful indicator of the extent of the phenomenon among the youth population. Many drugs of abuse are included in the WADA (World Anti-Doping Agency) Prohibited List, and are therefore routinely tested for in antidoping controls.

This study presents the data obtained in tests carried out in the period 20032007 at the Antidoping Laboratory of Rome, on 44781 samples analysed. The methods used are those developed by the Laboratory for routine antidoping analyses.

The percentage of positive test results ranges from 1.1 to $2 \%$, with a high incidence of stimulants and drugs of abuse. The substance most frequently found is THC metabolite, which accounts for $0.2-0.4 \%$ of the total, followed by cocaine metabolites, accounting for $0.1 \%$. Other stimulants found are ephedrines, carphedon, modafinil, and anorexic compounds such as phendimetrazine and norfenfluramine. No amphetamines or amphetaminelike designer drugs have been detected.

These data may be indicative of the widespread incidence of cocaine and cannabis abuse among the young Italian population, bearing in mind that the phenomenon is underestimated in this study, due to the fact that drugs of abuse are investigated only in samples involved in competitive sport, and especially to the fact that people doing such activities take more care of their health and are subject to greater control in their behaviour and habits with respect to the "normal" population.

Key words: urine analysis, drugs of abuse, antidoping controls, epidemiology. 


\section{INTRODUCCIÓN}

L a incidencia de consumo de sustancias de abuso en el deporte puede ser un indicador útil de la difusión del fenómeno en la población juvenil. Muchas drogas han sido incluidas en la lista de sustancias prohibidas de la WADA (World Anti-Doping Agency) ${ }^{1}$ entre los estimulantes están prohibidas las anfetaminas y los compuestos anfetaminosimiles, la cocaína, la efedrina, sustancias con potencial de abuso tales como los anorexizantes y potentes estimulantes centrales como el modafinilo, carfedón, metilfenidato; y entre los narcóticos la heroína y metabolitos, la metadona, fentanilo y derivados, la mayor parte de los opiáceos morfino-similes, y tambien incluido el grupo de los cannabinoides. Tales sustancias están prohibidas solo en competición, porque pueden desarrollar un efecto positivo sobre la prueba atlética solo si son consumidas en su proximidad.

Todas estas sustancias son investigadas de rutina sobre muestras analizadas junto a otras clásicas sustancias prohibidas como los agentes anabolizantes, las hormonas peptídicas, los beta-2 agonistas, los diuréticos y los glucocorticosteroides. La lista se revisa por lo menos anualmente para incluir nuevas sustancias. Este estudio muestra los datos obtenidos en los controles efectuados de rutina en el laboratorio antidoping de Roma durante el periodo 2003-2007.

\section{MÉTODOS}

Este estudio se refiere a la temporada 2003-2007. Los análisis se efectuaron sobre un total de 44781 muestras, recogidas durante y fuera de la competición. Las muestras se analizaron con las metodologias desarrolladas por el laboratorio para la determinación de todas las sustancias incluidas en la lista prohibida.

En particular, para la determinacion de los estimulantes y drogas de abuso, las muestras positivas en los análisis de screening preliminar han sido confirmadas con cromatografía de gases/espectrometria de masas (GC/MS). Las sustancias cuya determinación está sometida a un punto de corte (catina, efedrinas, metabolito del cannabis y morfina) han sido cuantificadas y solo las que superaban el umbral han sido incluidas entre los positivos. Los valores del punto de corte son $15 \mathrm{ng} / \mathrm{ml}$ para el metabolito ácido del THC, $1 \mu \mathrm{g} /$ $\mathrm{ml}$ para la morfina, $5 \mu \mathrm{g} / \mathrm{ml}$ para la catina y $10 \mu \mathrm{g} / \mathrm{ml}$ para la efedrina. La sola presencia de todos los otros estimulantes, sin umbral, está prohibida.

\section{RESULTADOS}

El tanto por ciento de positivos encontrados en el periodo 2003-2007 en el laboratorio de Roma varía entre el 1,1 y el $2 \%$ con una elevada incidencia de uso de sustancias estimulantes y drogas de abuso. La sustancia más frecuentemente encontrada es el metabolito del cannabis, representando el $20 \%$ respecto a las otras sustancias y de un $0,2-0,4 \%$ del total de las muestras analizadas. También se detecta frecuentemente la presencia de cocaína, con una incidencia de positividad alrededor de un $10 \%$ y del $0,1 \%$ respecto al total. Los casos positivos a efedrina han descendido en los últimos años, así mientras en el año 2003 eran cercanos al $20 \%$ en el 2007 no llegan a suponer ni un 3\% de los casos, y menor incidencia se observa aún en el uso de anorexizantes (concretamente solo se encontraron dos casos de fendimetrazina y uno de norfenfluramina) y ningún caso de anfetaminas o compuestos anfetaminosimiles. Se detectaron dos casos positivos a carfedon, uno de ellos relativo a un atleta del Este durante las Olimpiadas invernales de Turín de 2006 y tres casos de modafinilo. No se ha detectado ningún caso de narcóticos salvo dos casos positivos a morfina, pero que era compatible con procesos metabólicos de la codeína. En las figuras 1-5 se muestran los datos de positividades encontrados cada año. En las figuras 6 y 7 se muestra la evolución de la positividad a las drogas de abuso, y se puede apreciar como subió tanto el consumo de cocaína como el de cannabis, mientras que no se encontraron drogas de diseño.

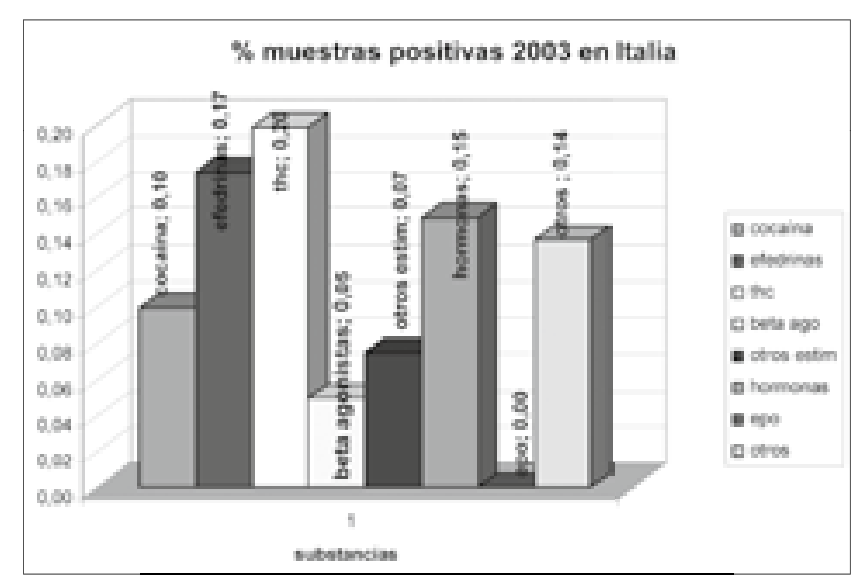

Figura 1. Positivos encontrados en 2003 (muestras totales $\mathbf{= 8 0 5 2}$ ).

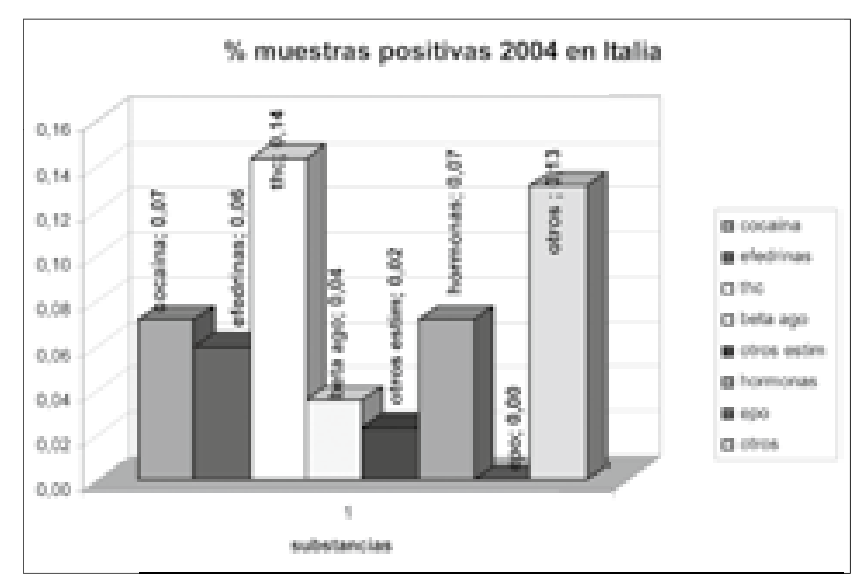

Figura 2. Positivos encontrados en 2004 (muestras totales analizadas $\mathbf{= 8 3 6 6}$ ). 


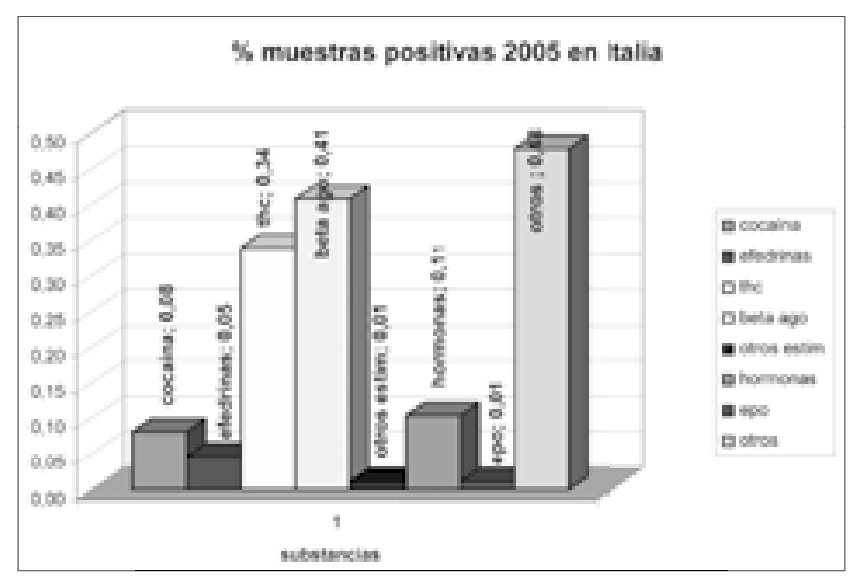

Figura 3. Positivos encontrados en 2005

(muestras totales analizadas $\mathbf{= 8 5 4 3}$ ).

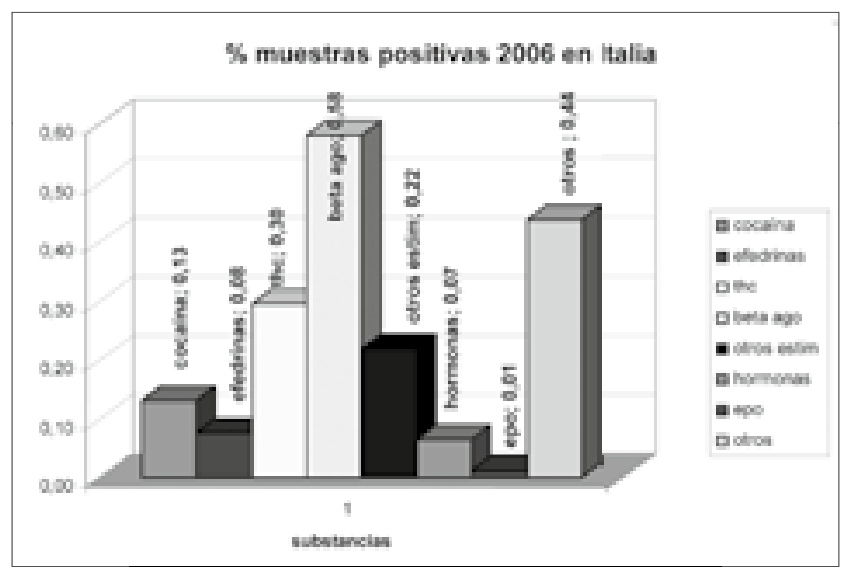

Figura 4. Positivos encontrados en 2006 ( muestras totales analizadas $=9120$ ).

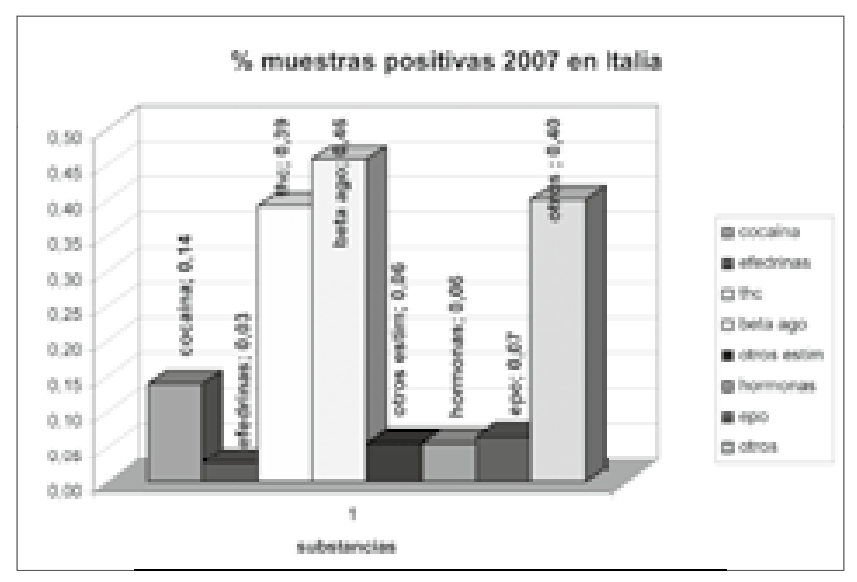

Figura 5. Positivos encontrados en 2007 ( muestras totales analizadas $=10700$ ).

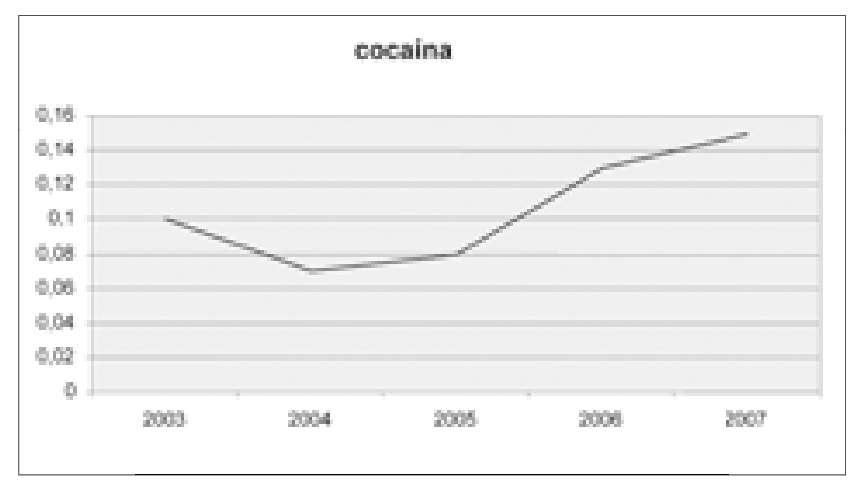

Figura 6. Evolución del número de positivos para metabolitos de la cocaína en Italia.

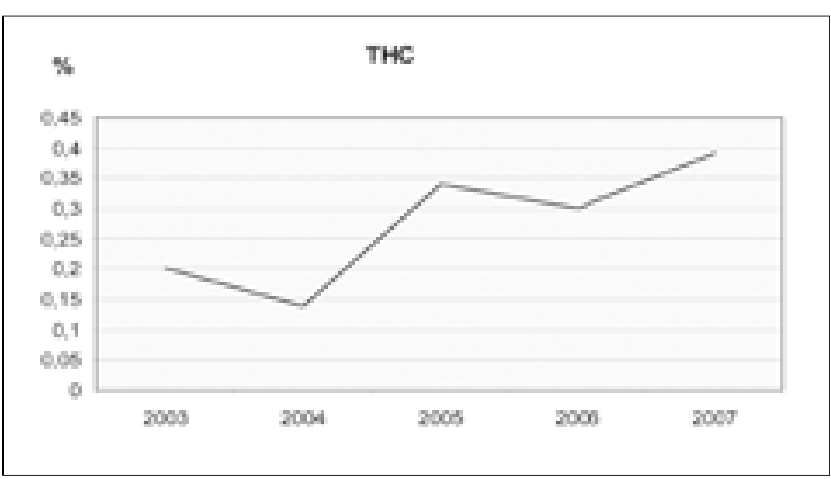

Figura 7. Evolución del número de positivo para el metabolito del cannabis en Italia.

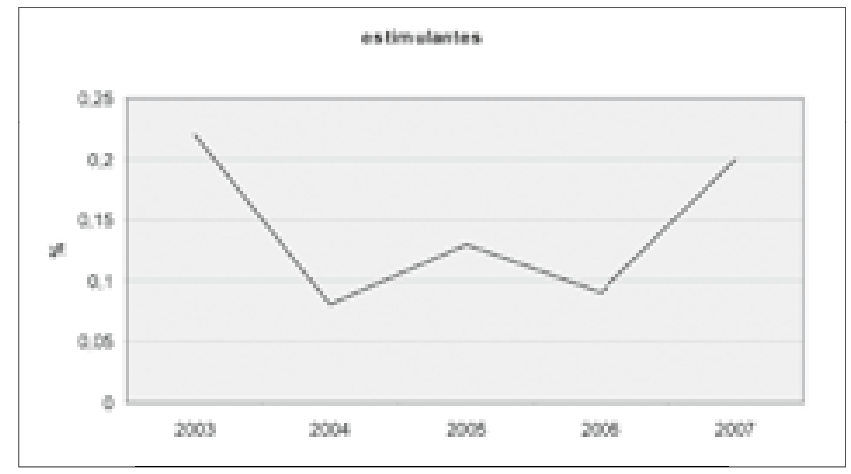

Figura 8. Evolución del número de positivos para estimulantes en España.

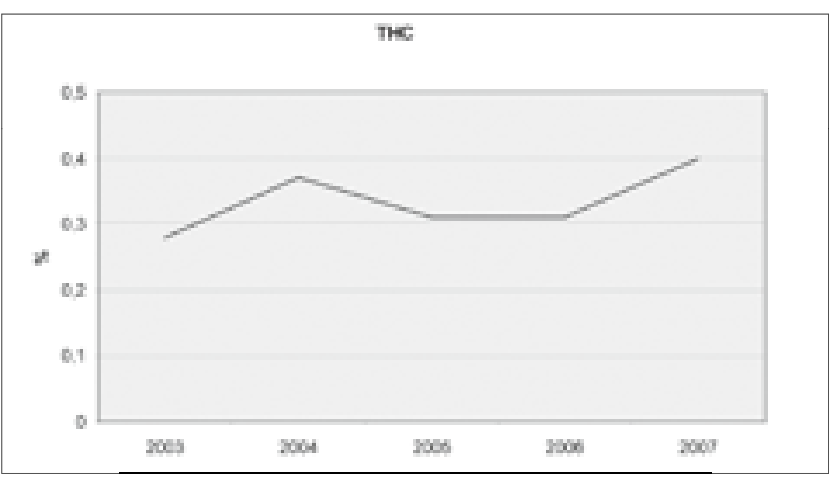

Figura 9. Evolución del número de positivos para metabolitos del cannabis en España. 


\section{DISCUSIÓN}

Estos datos pueden ser un indicador útil de la elevada incidencia del uso de cannabinoides, ya predecible, y también de cocaína por la población juvenil, considerando una infraestimación del fenómeno ya que, por una parte, estos datos se refieren solo a controles efectuados durante competición y que valores inferiores al punto de corte no son claramente considerados positivos; y por otra parte, a la propia población estudiada, atletas los cuales están sometidos a controles de hábitos de salud y forma física, y que saben que están sometidos a controles antidoping, cosas que no ocurren con jóvenes de la misma edad que no practican deporte de competición.

Las tendencias encontradas, muestran un aumento de consumo tanto de cannabis como de cocaina en los años estudiados, al igual que ocurre con los datos publicados por la Dirección Central de Servicios Antidroga (DCSA) sobre la evolución de las drogas decomisadas en los ultimos diez años en Italia, donde se pudo observar una tendencia constante de los alijos de cannabis y un aumento de los de cocaina ${ }^{2}$.

La misma tendencia se observa en España, tanto en la tipologia de los alijos secuestrados, como está descrito en las estadisticas del Plan Nacional Sobre Drogas ${ }^{3}$, como en los porcentajes de muestras positivas a drogas en los controles antidopaje efectuados en estos años (estadisticas anuales de la WADA) ${ }^{4}$.

En la literatura internacional se encuentran revisiones que indican que tanto los estimulantes como el cannabis son drogas que pueden mejorar las capacidades deportivas tanto durante la competiciòn, como durante el entrenamiento, los primeros por su capacidad de aumentar los efectos (directos o indirectos) de los neurotransmisores adrenérgicos, y el segundo por su efecto relajante ${ }^{5-10}$. De todas maneras, aparte de algunos casos de anorexigenos, las tipologia de sustancias encontradas (principalmente cocaína y cannabis), y el hecho que en la mayoria de los casos de cocaína se encuentren solo sus metabolitos, indicando un abuso de la droga alejado de la competición, hacen suponer una utilización mayormente social de estas sustancias, mas que por fines de dopaje. Entre la población juvenil hay la tendencia a considerar el cannabis como una droga que puede mejorar el rendimiento, pero en la mayoria de los casos las concentraciones encontradas en orina son compatibles con restos de consumos ocurridos unos días antes de la prueba (fase de eliminación tardía). Por el contrario, en el caso de las efedrinas, aunque son el principio activo de muchos medicamentos, su utilización en el deporte parece ser debida mayormente a su actividad estimulante, siendo utilizadas principalmente en algunas competiciones, en dosis elevadas y generalmente no siendo declaradas.

La reflexión que se debe hacer es que estas sustancias, especialmente el cannabis, aunque no sean consumidas para mejorar la forma física o la competición, sino por una costumbre personal o para relajarse antes de la prueba, están de todas maneras incluidas en la lista de las sustancias prohibidas y su uso no está justificado por dos motivos fundamen- tales: la tutela de la salud de los atletas ${ }^{11,12}$ y la necesidad de que la imagen de los deportistas, que son a menudo ídolos para los jóvenes, sea un ejemplo positivo y limpio.

\section{REFERENCIAS}

The World Anti-doping Code Prohibited list International Standard. Disponible en: http://www.wada-ama.org. [Consultado el 31/12/2008]

Statistiche annuali Direzione Centrale Servizi Antidroga (DCSA). Disponible en: http://www.poliziadistato.it [Consultado el 31/12/2008].

Informe 2007 del observatorio español sobre drogas. Situación y tendencias de los problemas de drogas en españa Disponible en: http://www.pnsd.msc.es/ [Consultado el 31/12/2008].

Wada Laboratory Statistics. Disponible en: http:// www.wada-ama.org. [Consultado el 31/12/2008]

Docherty JR. Pharmacology of stimulants prohibited by the World AntiDoping Agency (WADA). Br J Pharmacol 2008; 154: 606-22.

Avois L, Robinson N, Saudan C, Baume N, Mangin P, Saugy M. Central nervous system stimulants and sport practice. $\mathrm{Br} J$ of Sports Med 2006; 40: 16-20.

Lorente FO, Peretti-Watel P, Grelot L. Cannabis use to enhance sportive and non-sportive performances among French sport students. Addict Behav 2005; 30: 1382-91

Saugy M, Avois L, Saudan C, Robinson N, Giroud C, Mangin P, Dvorak J. Cannabis and Sport. Br J Sports Med 2006; 40: 13-15.

Moore MJ, Werch CE. Sport and physical activity participation and substance use among adolescents. J Adolesc Health 2005; 36 486-93.

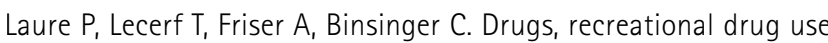
and attitudes towards doping of high school athletes. Int J Sports Med 2004; 25: 133-8.

Ramos Gordillo AS. Lucha contra el dopaje como objetivo de salud. Adicciones 1999; 11: 299-310.

Valero Valenzuela A, Ruiz Juan F, García Montes, ME, Granero Gallegos A, Martínez Rodríguez AM. Relación entre la práctica de actividad físico-deportiva y el consumo de alcohol de los ciudadanos de más de 14 años. Adicciones 2007; 19: 239-250. 